journal club

\title{
Auch chronisch neuropathische periphere Schmerzen werden gelindert
}

Fragestellung: Ist die lokale Injektion von Botulinumtoxin bei chronisch neuropathischen Schmerzen nach und bei peripherer Nervenschädigung wirksam?

Hintergrund: Botulinumtoxin A ist seit langem zur Behandlung von Dystonien und seit einiger Zeit auch zur Behandlung der Spastik zugelassen. Eine weitere Zulassung besteht für die chronische Migräne. Die klinische Erfahrung in der Vergangenheit hatte immer wieder gezeigt, dass Patienten bei denen eine Dystonie im Rahmen einer peripheren Nervenschädigung auftrat, nicht nur über eine Reduktion der dystonen Muskelbewegungen und -krämpfe berichteten, sondern auch über eine Reduktion ihrer Schmerzen. Physiologischer Hintergrund der Wirkung von Botulinumtoxin ist in diesen Fällen offenbar eine Bindung an periphere C-Fasern. Kleinere Studien bei neuropathischen Schmerzen wie beispielsweise der postzosterischen Neuralgie hatten ebenfalls eine Wirksamkeit von Botulinumtoxin A gezeigt. Sorgfältig geplante und durchgeführte randomisierte placebokontrollierte Studien gab es aber bisher nicht.

Patienten und Methodik: Die doppelblinde, placebokontrollierte Studie schloss Patienten mit chronisch neuropathischen Schmerzen mit einem mittleren Schmerzscore von mindestens 4 auf einer Skala von 0 bis 10 und einer Schmerzdauer von mindestens sechs Monaten ein.

Attal N, de Andrade DC, Adam $F$ et al. Safety and efficacy of repeated injections of botulinum toxin A in peripheral neuropathic pain (BOTNEP): a randomised, double-blind, placebocontrolled trial. Lancet Neurol 2016; 15: 555-65
Die meisten Patienten hatten eine traumatische oder chirurgisch verursachte periphere Nervenschädigung. Die Patienten erhielten entweder zwei subkutane Gaben von Botulinumtoxin im schmerzhaften Areal mit bis zu 300 Einheiten oder Placebo im
Abstand von zwölf Wochen. Der primäre Endpunkt war die Änderung verglichen mit der Baseline der vom Patienten berichteten mittleren wöchentlichen Schmerzintensität über einen Zeitraum von 24 Wochen nach der ersten Injektion.

Ergebnisse: Insgesamt wurden 68 Patienten randomisiert. Für die Endauswertung in der Intention-to-treat-Analyse standen die Ergebnisse von 34 Patienten in der Botulinumtoxin-A- und von 32 Patienten in der Placebogruppe zur Verfügung. Die Patienten waren im Mittel 52 Jahre alt, die Hälfte waren Männer. Bei $45 \%$ war der Schmerz im Bereich der oberen, bei $37 \%$ an der unteren Extremität und bei den übrigen am Rumpf lokalisiert. Die mittlere Dauer der chronischen Schmerzen betrug 5,7 Jahre und die mittlere Schmerzintensität auf einer Skala von 0 bis 10 betrug 6,5. Zwei Drittel der Patienten waren mit trizyklischen Antidepressiva vorbehandelt und 30\% mit Gabapentin oder Pregabalin, 50\% nahmen Opioide ein. Die mittlere Schmerzintensität in der Baseline betrug in der Botulinumtoxin-AGruppe 6,5 und nach 24 Wochen 4,6. In der Placebogruppe war die Schmerzintensität bei Baseline 6,4 und am Ende der Behandlung 5,8. Der Unterschied zwischen Botulinumtoxin A und Placebo war signifikant. Der Therapieeffekt war bereits nach zwei Wochen nachweisbar und hielt über 24 Wochen an. Bei den sekundären Zielparametern waren paroxysmale einschießende Schmerzen und die Allodynie ebenfalls unter Botulinumtoxin A signifikant gebessert. Auf einer Angstskala fand sich eine signifikante Besserung, die Depressionsskala war unverändert. Auch die Schlafqualität besserte sich unter Botulinumtoxin A.

Schlussfolgerungen: Zwei lokale Injektionen von Botulinumtoxin A bei Patienten mit chronisch neuropathischen, durch eine periphere Nervenläsion bedingten Schmerzen sind einer Behandlung mit Placebo überlegen. Eine Allodynie scheint einen prädiktiven Wert für den Therapieerfolg zu haben.

\section{- Kommentar von Hans-Christoph Diener, Essen}

\section{Für bestimmte Patienten durchaus zu erwägende Off-label-Therapie}

Dies ist die erste große, gut geplante und durchgeführte Studie zum therapeutischen Einsatz lokaler Botulinumtoxin-A-Injektionen bei chronisch neuropathischen Schmerzen. Der Therapieunterschied ist in absoluten Zahlen nicht sehr groß, aber signifikant. Dabei muss berücksichtigt werden, dass die Patienten bereits seit mehr als fünf Jahren unter den chronisch neuropathischen Schmerzen litten und eine gleichzeitig bestehende kombinierte medikamentöse Schmerztherapie offenbar nicht ausreichend wirksam war. Der große Vorteil von Botulinumtoxin A ist, dass keine zentralen Nebenwirkungen auftreten. Es werden von den Patienten lediglich Schmerzen an der Injektionsstelle geklagt. Die Autoren haben auch berechnet, wie sich ihre Studie in die bisherigen Metaanalysen, die Numbers needed to treat (NNT) ermittelt haben, einfügt. Die NNT in dieser Studie betrug 7,3 verglichen mit Pregabalin mit 7,7, starken potenten retardierten Opioiden mit 4,3 und trizyklischen Antidepressiva mit 3,6. Derzeit handelt es sich bei der lokalen Anwendung von Botulinumtoxin noch um eine Off-label-Indikation. Sie ist aber erwägenswert bei Patienten, die eine medikamentöse Therapie wegen Nebenwirkungen nicht tolerieren, oder bei denen Opioide wegen einer hohen Suchtgefahr nicht eingesetzt werden können. 